УДК 532.546:536.24:004.942

\title{
CFD-МОДЕЛИРОВАНИЕ СОПРЯЖЕННОГО ПАРО- И ВЛАГОПЕРЕНОСА В ПОРИСТЫХ СТРОИТЕЛЬНЫХ МАТЕРИАЛАХ
}

Круковский П.Г., докт. техн. наук, Полубинский А.С., канд. физ-мат. наук, Тадля О.Ю., канд. техн. наук, Коваленко В.Н., канд. техн. наук

Институт технической теплофизики НАН Украинь,, ул. Желябова, 2a, 03680, Украина

Розглянуто CFD-модель, що враховує процеси переносу пари та вологи, що надходять в пористі будівельні матеріали 3 вологого повітря. На відміну від існуючих, розглянута модель, яка враховує спряжений паро- та вологоперенос, а також співвідношення між паровою та вологістною складовими відповідно до кривої сорбції, наявної для розглянутого будівельного матеріалу. Проведено аналіз особливостей реалізації розробленої моделі в середовищі програми ANSYS-FLUENT, а також порівняння результатів розрахунків 3 експериментальними даними при зволоженні газобетону.
Рассмотрена CFD-модель, учитывающая процессы сопряжённого переноса пара и влаги, поступающие в пористые строительные материалы из влажного воздуха. В отличие от существующих, рассмотренная модель учитывает сопряженный паро- и влагоперенос, а также соотношение между паровой и влажностной составляющими в соответствии с кривой сорбции, имеющейся для рассматриваемого строительного материала. Проведен анализ особенностей реализации разработанной модели в среде программы ANSYS-FLUENT и сравнение результатов расчетов с экспериментальными данными при увлажнении газобетона.
Presented CFD-model considers coupled vapor and moisture transfer processes in porous building materials. In contrast to the existing models, it takes into account the ratio between vapor and moisture components in accordance with the building materials' sorption curve. The specific analysis of the model development in ANSYSFLUENT is presented and the results are compared with experimental data of the gas concrete humidification.

Библ. 12 , табл. 1 , рис. 5.

Ключевые слова: пористые строительные материалы, паро- и влагоперенос, кривая сорбции, CFDмоделирование.

$a$ - соотношение между влагосодержанием и абсолютной влажностью согласно кривой сорбции для данного материала;

$B$ - барометрическое давление, 101325 Па;

$D$ - коэффициент диффузии пара в воздухе, $\mathrm{m}^{2} / \mathrm{c}$;

$D_{w}$ - влагопроницаемость (агрегатное состояние вода) в материале, кг/(м·c·\%);

$d$ - содержание влаги в каждой ячейке, кг/кг;

$H$ - энтальпия, кДж/кг;

$h_{v}$ - теплота парообразования, 2,5·10 Дж/кг;

$k$ - степень интенсивности поглощения (либо выделения) влаги из пара паровоздушной смеси; $m$ - концентрация (доля паров воды во влажном воздухе), кг/кг;

$P$ - парциальное давление, Па;

$P_{\text {нас }}$ - давление насыщения, Па;

В течение последних десятилетий проектируются и строятся здания высокой энергоэф- $w$ - влагосодержание в материале, кг/кг;

$x$ - координата, м;

$\beta$ - коэффициент массоотдачи, кг/( $\mathrm{M}^{2} \cdot \mathrm{c} \cdot$ Па);

$\delta$ - паропроницаемость (агрегатное состояние пар) материала, кг/(м·с·Па);

$\lambda_{w}$ - теплопроводность влажного материала,

$\mathrm{BT} /(\mathrm{M} \cdot \mathrm{C})$

$\tau$ - время, с.

\section{Нижние индексы:}

v - параметр относится к пару;

w - параметр относится к влаге;

Dry - свойство, относящееся к сухому материалу; нар - параметр относится к области снаружи газобетона;

пов - параметр относится к границе.

фективности. Эти здания должны проектироваться без ущерба для комфорта, находящих- 
ся в них людей. Два ключевых параметра: температура и относительная влажность воздуха, играют важную роль в определении условий в помещениях и зданиях. В то время как влияние температуры хорошо известно и изучено, влияние влажности не всегда принимается во внимание. Необходимо учитывать изменение влажностного режима, как в самом помещении, так и внутри ограждения для того, чтобы не допускать конденсации влаги на внутренней поверхности наружных ограждений и/или внутри их, поскольку повышенная или пониженная влажность в помещении может сделать некомфортной жизнь жителей, а также вызывать серьезные проблемы со здоровьем у жителей из-за наличия плесени и грибка.

Теплозащитные свойства ограждающих конструкций существенно зависят от влажностного состояния материалов, повышение которой приводит к снижению их теплозащитных качеств (за счет увеличения теплопроводности ограждающих конструкций), увеличению теплопотерь и снижает их долговечность.

Анализ и прогнозирование влажности ограждающих конструкций можно осуществлять с помощью моделирования сопряженного парои влагопереноса между помещением и этими конструкциями. Для этого модели должны учитывать раздельно составляющие в виде пара (в помещении и ограждающей конструкции) и влаги (в ограждающей конструкции).

Существуют различные методы моделирования влажностных задач. Упрощенные инженерные методики используются для оценочных расчетов количества влаги, которое может накопиться в ограждающей конструкции, мест возможного возникновения конденсата на поверхности или внутри конструкции (т.н. «точки росы») [1-3]. Они имеют следующие основные недостатки - использование стационарного теплового режима, как помещения, так и окружающей среды, а также постоянных значений коэффициентов материалов (паропроницаемости, влагопроницаемости и т.п.). Эти упрощения не позволяют получать реальную картину текущего тепловлажностного состояния в ограждающей конструкции.

С появлением производительной компью- терной техники, разработанные инженерные зависимости стали широко применяться в программах различного уровня сложности, краткое сравнение возможностей которых приведено в [4]. Обычно эти программы рассчитывают средние температуры и относительные влажности в помещении и большинство из них не учитывают перенос массы пара на границе между воздухом и ограждающей конструкцией здания.

Поскольку использование средней температуры воздуха для различных потребностей расчетов теплового состояния помещения было недостаточно, стали развиваться методы CFDмоделирования. Эти модели наиболее полно описывают процессы тепло- и влагообмена, происходящие в помещении и стенах. Однако на текущий момент есть технические сложности в реализации полного сопряженного тепло- и влагообмена для помещения [5]. Проблема в данном случае связана еще и с тем, что для полного сопряженного теплообмена с учетом радиационной составляющей ограждающие конструкции помещения должны представлять собой твердые стены (в программе ANSYS-FLUENT необходимо задавать опцию «wall»). Однако, в таком случае невозможно передавать поток массы через стены (перенос пара, влаги, воздуха в окружающую среду или из нее). Для того, чтобы решить эту проблему пользователи CFD-программ вынуждены писать специальные подпрограммы, которые носят название «user define function» или же «user define scalar».

Поскольку решение полностью сопряженной нестационарной задачи тепломассобмена в помещении с ограждениями было сложной задачей, некоторые исследователи пошли по пути соединения CFD-модели помещения и упрощенной влажностной модели в сосредоточенных параметрах ограждения [6]. Однако, такой подход не позволяет решать задачу движения и накопления влаги в конструкции. Как один из вариантов решения проблемы, существует подход по представлению стены как неподвижной жидкости с соответствующими свойствами материалов. Этот подход использован в [7]. В этой модели решалась стационарная задача и не учитывался радиационный теплообмен 
между поверхностями ограждений помещения, и не было сравнения с данными натурного эксперимента.

В процессе паро- и влагопереноса через ограждения участвуют три вида веществ: воздух, пар и жидкость. При этом в ограждающей конструкции происходит диффузия водяного пара в движущейся паровоздушной смеси через поры материала ограждения, - фильтрация влажного воздуха из-за разницы влажностей окружающей среды и внутреннего воздуха, а также возникновение и перемещение влаги в виде адсорбируемой жидкости, приводящей к изменению влагосодержания материала ограждения. Эти процессы не действуют раздельно, а оказывают друг на друга взаимное влияние. Увеличение влагосодержания материала ограждения ведет за собой уменьшение относительной влажности паровоздушной смеси, которая отдает часть влаги материалу ограждения. Увеличение влажности материала ограждения называется сорбцией, снижение влажности материала называется десорбцией материала. Зависимость между количеством влаги в материале и влажностью воздуха, находящегося в виде паровоздушной смеси в материале, называют изотермой сорбции. Изотермы определяются экспериментальным путем для каждого материала при комнатной температуре.

Для прогнозирования изменения влажностного состояния ограждающих конструкций в помещении необходимы кривые сорбции материалов конструкции и эмпирические коэффициенты их паро- и влагопроницаемости. Однако, приведенные в литературе для каждого материала параметры, не могут широко использоваться, поскольку определяются экспериментальным путем для конкретных условий и ограниченных значений различных параметров (температура, влагосодержание, пористость материала и относительная влажность). Это же касается коэффициента массоотдачи между влажным воздухом и конструкций $\beta$, численное значение которого определяется в зависимости от коэффициента конвективной теплоотдачи, значения не всегда точно известного. Также возникает необходимость в проведении долговре- менных расчетов, которые позволяют расчетным путем определить время и степень насыщения влагой материалов конструкции.

Проведенный литературный поиск [4] показал отсутствие среди существующих моделей, учитывающих отдельно паровую и влажностную составляющие. Также нет данных о проверке получаемых результатов на удовлетворение соотношения пара в воздухе и влаги в исследуемом материале в стационарном режиме. Поэтому разработка модели, учитывающей сопряженный паро- и влагоперенос между воздушной средой и строительной конструкцией помещения с учетом пара и влаги отдельно является актуальной задачей, решаемой ниже авторами.

Ставится задача разработать модель, которая будет удовлетворять следующим требованиям:

1. модель тепловлагопереноса должна удовлетворять соотношению влаги в материале ограждающих конструкций и пара согласно кривой сорбции для этого материала при долговременном расчете;

2. модель должна описывать нестационарное распределение пара и влаги (отдельно) в объеме строительного материала;

3. модель должна учитывать переход пара из объема помещения в ограждающую конструкцию и его переход во влагу и наоборот в самой ограждающей конструкции;

4. модель должна быть сопоставлена с имеющимися экспериментальными данными.

5. модель должна выполнять расчеты теплопаро- и влагопереноса в материале в широко используемых компьютерных CFD-программах, например, ANSYS-FLUENT.

Будем различать стационарный равновесный процесс переноса пара и влаги и нестационарный неравновесный процесс в материале. Первый определяется кривой сорбции, в то время как во втором соотношение пара и влаги может быть не равным соотношению по кривой сорбции.

Коэффициент паропроницаемости представляет собой количественную величину потока пара, проходящего через материал толщиной 1 м за единицу времени при единичной разнице парциальных давлений. 
Коэффициент влагопроницаемости характеризует количество влаги за единицу времени через единицу длины при определенных условиях влажности на поверхностях.

Два вышеприведенных коэффициента определяются в стационарных тепловых и влажностных условиях на образцах материалах и на наш взгляд не могут служить характеристиками нестационарного процесса увлажнения или осушения материала. Введем коэффициент $k$, который характеризует интенсивность поглощения (выделения) влаги из (в) паровоздушную смесь в нестационарных условиях.

Целью работы является разработать и верифицировать CFD-модель, учитывающую процессы сопряженного переноса пара и влаги в пористых строительных материалах, и отвечающую всем вышеперечисленным требованиям.

Рассмотрим процессы переноса влаги и пара в пористых строительных материалах на примере увлажнения образца газобетона размером 50×50×100 мм [10]. Созданная модель тестировалась (согласно требованиям п.5.) по результатам экспериментальных исследований влагосодержания по высоте блока газобетона, приведенным в [10]. В экспериментальной установке предварительно высушенные образцы помещались в изолированные камеры, где снизу поддерживались постоянные температурные и влажностные условия $\left(70{ }^{\circ} \mathrm{C}\right.$ и $\left.100 \%\right)$, а сверху $-70{ }^{\circ} \mathrm{C}$ и $0 \%$. Замеры влажности в блоке проводились в различные моменты времени в шести местах по высоте блока.

Физическая модель процессов, происходящих в исследуемом образце следующая. Молекулы пара и влаги из воздуха нижней камеры абсорбируются нижней поверхностью сухого блока (поверхностная сорбция). По условиям эксперимента в нижней камере постоянно присутствуют пар и влага, которые являются источником при увлажнении газобетона. Далее по высоте блока пар и влага в паровоздушной смеси перемещаются вверх по порам и капиллярам. Движения самой паровоздушной смеси в образце не учитывается. По пути паровоздушная смесь осушается (уменьшается количество пара) за счет того, что она отдает материалу блока часть в виде влаги. Некоторая часть вла- ги также конденсируется на поверхности пор, превращаясь в капли воды. При наличии определенных условий сконденсированная влага и влага в материале может возвращаться обратно из материала в паровоздушную смесь. Максимальное содержание влаги в материале газобетона ограничивается значением его сорбционной влажности. Дальнейшее накопление влаги в области, где достигнуто максимальное влагосодержание, не происходит. В этом случае, увеличивается влагосодержание рядом расположенных областей.

Поскольку в пористых материалах ограждений влага может перемещаться в парообразной и/или жидкой фазе из-за различных движущих сил, то большинство ученых принимает два или более потенциалов для переноса влаги (парциальное давление и влагосодержание). Рассмотрим ряд моделей.

Модель 1. Математическая модель процессов тепло-, паро- и влагопереноса согласно [8] в ограждающей конструкции описывается двумя уравнениями.

- Уравнением паро- и влагопереноса в материале ограждающей конструкции

$$
\frac{\partial w}{\partial \tau}=\frac{\partial}{\partial x}\left[D_{w} \frac{\partial w}{\partial x}\right]+\frac{\partial}{\partial x}\left[\delta \frac{\partial P}{\partial x}\right],
$$

где первое слагаемое в правой части отвечает за транспорт воды в материале, второе - за транспорт пара в материале, а левая часть это количество влаги, которая накапливается в материале.

- Уравнением энергии для всей области решения

$$
\frac{\partial H}{\partial \tau}=\frac{\partial}{\partial x}\left[\lambda_{w} \frac{\partial T}{\partial x}\right]+h_{v} \frac{\partial}{\partial x}\left[\delta \frac{\partial P}{\partial x}\right],
$$

где первое слагаемое в правой части отвечает за передачу теплоты во влажном материале, второе - за влияние теплоты парообразования/конденсации на тепловой режим ограждения.

В явном виде соотношение между паром и влагой согласно кривой сорбции в данной модели не учитывается. Это не позволяет остановить процесс накопления влаги материалом, т.к. отсутствует величина максимального влагосодержания известная из изотермы сорбции. Поскольку 
в модели нет отдельно паровой и влажностной составляющей, решение сопряженной задачи с воздухом невозможно.

Также стоит обратить внимание на то, что входящие в уравнение коэффициенты $D_{w}$ и $\delta$ находятся экспериментально в стационарных равновесных условиях и для условий нестационарных расчетов, строго говоря, применяться не могут. Следовательно, их необходимо идентифицировать по результатам имеющихся экспериментов.

Добавим к системе из двух предыдущих уравнений третье, отвечающее за диффузию пара в паровоздушной смеси (воздухе). Это позволит решать задачу тепло- и влагопереноса как в самом ограждении, так и в паровоздушной среде внутреннего объема помещения.

$\frac{\partial m}{\partial \tau}=\frac{\partial}{\partial x}\left[D \frac{\partial m}{\partial x}\right]$

Парциальное давление в формуле (1) определяется

$$
P=\frac{d \cdot B}{0,622+d},
$$

где $d=m /(1-m)$ - концентрация влаги в рассматриваемом объеме.

Под коэффициентом $D$ поднимается коэффициент диффузии пара в паровоздушной среде в порах газобетона и численно он не равен коэффициенту диффузии пара в воздухе. Система уравнений (1) - (2) широко распространена и используется $[6,8,9]$ для определения общего количества пара и влаги в материале без различия этих двух компонентов.

Рассматривая влагу, как часть паровоздушной смеси, которая проникает внутрь пористого строительного материала, авторы подразумевают под относительной влажностью содержание пара и влаги в смеси, а под влагосодержанием количество только влаги в материале.

Модель 2. Авторами предлагается моделирование перехода части пара во влагу через некий коэффициент $k$, который говорит о том, что при $m>w$ из свободной паровоздушной смеси часть влаги поглощается пористым материалом, и наоборот, при $w>m$ влага возвращается в смесь из материала. Назовем $k$ коэффициентом интенсивности поглощения (выделения) влаги из (в) паровоздушной смеси. В модели должен соблюдаться баланс между коэффициентом $k$ и $D_{w}$. Если коэффициент $k$ имеет небольшое значение, это означает, что в модели имеется небольшое количество влаги, за транспорт которого отвечает $D_{w}$. Т.о., мы заменяем слагаемое со второй производной по парциальному давлению в уравнении (1) слагаемым с коэффициентом $k$, численно определяющим количество влаги из смеси переходящее в пористый материал.

Тогда уравнение (1) можно записать в виде

$$
\frac{\partial w}{\partial \tau}=\frac{\partial}{\partial x}\left[D_{w} \frac{\partial w}{\partial x}\right]+\frac{\partial}{\partial x}\left[k \cdot(a \cdot m-(w+m)) \frac{\partial P}{\partial x}\right]
$$

где $k \cdot(a \cdot m-(w+m)) \frac{\partial^{2} P}{\partial x^{2}}-$ слагаемое, отвечающее за количество пара, переходящее во влагу или наоборот (условно назовем его источник), в котором:

$k \cdot(a \cdot m-(w+m))-$ степень поглощения/накопления влаги, а $\frac{\partial^{2} P}{\partial x^{2}}-$ сомножитель, отвечающий за транспорт пара.

Коэффициент $a=(w+m) / m$ в (5) отвечает за соотношение влаги и пара в материале согласно кривой сорбции, как в стационарном, так и нестационарном режимах. Когда $w+m=a * m$, тогда поглощения/накопления влаги нет. При этом транспорт пара есть, если $\partial^{2} P / \partial x^{2} \neq 0$. Накопление может быть в соседних ячейках, где $w+m \neq a^{*} m$. Интенсивность транспорта пара, определяемого величиной $\partial^{2} P / \partial x^{2}$ и интенсивность насыщения пара $k \cdot(a \cdot m-(w+m))$ могут независимо приводить к обнулению источника. Максимальное значение $w+m$ в любой точке в стационарном режиме (для бесконечного момента времени) должно соответствовать кривой сорбции при своем значении $m$. Если $m>w$, то тогда второе слагаемое будет иметь отрицательное значение и будет происходить возврат влаги в пар.

Тогда в уравнении (3) необходимо отнять некоторое количество влаги, которое образуется из пара паровоздушной смеси.

$$
\frac{\partial m}{\partial \tau}=\frac{\partial}{\partial x}\left[D \frac{\partial m}{\partial x}\right]-\frac{\partial}{\partial x}\left[k \cdot(a \cdot m-(w+m)) \frac{\partial P}{\partial x}\right],
$$


где первое слагаемое в правой части отвечает за диффузию пара в паровоздушной смеси, втоpoe - за количество влаги, которая удаляется из паровоздушной смеси. В обеих моделях учитывается зависимость коэффициента теплопроводности влажного материала от влагосодержания

$\lambda(w)=\lambda_{\text {Dry }}+\lambda_{w} \cdot w$

Изотермы сорбции материалов определяются обычно при комнатной температуре весовым методом согласно [11] по нескольким экспериментальным измерениям (для газобетона см. рис. 1, а). Полученное в эксперименте путем взвешивания массовое влагосодержание по изотерме сорбции является суммой количества влаги и пара, которые находятся в образце, в то время как в разработанной модели эти две составляющие разделены. Поскольку в литературе не нашлось данных о том, как ведет себя кривая сорбции для газобетона при температуре $70{ }^{\circ} \mathrm{C}$, авторы статьи попытались самостоятельно оценить, как изменится кривая сорбции с повышением температуры.

В расчетах кривую сорбции для $20{ }^{\circ} \mathrm{C}$ нельзя использовать, т.к. абсолютная влажность, эквивалентная $100 \%$ относительной при температуре $20{ }^{\circ} \mathrm{C}$, равна 14 г/кг, в то время как при $70{ }^{\circ} \mathrm{C}$ эта же величина равна уже 210 г/кг. Оценив по $[9,10]$ приведенные результаты сорбционного увлажнения при 20 и $70^{\circ} \mathrm{C}$ авторы установили, что при значении времени 168 ч (максимальное значение времени для эксперимента

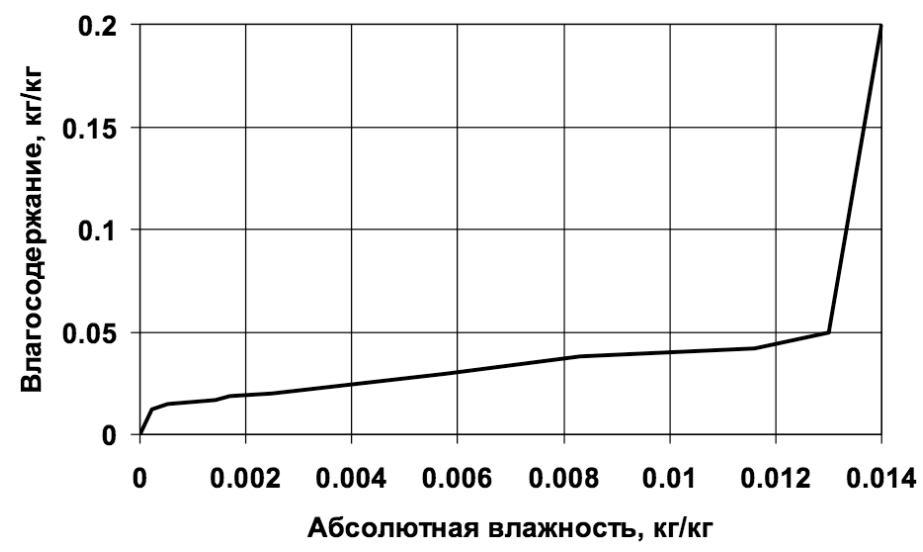

a с температурой $\left.70{ }^{\circ} \mathrm{C}\right)$ соотношение влагосодержаний при 70 и $20{ }^{\circ} \mathrm{C}$ в блоке газобетона равно 3,5. Заметим также, что угол наклона экспериментально определенной кривой сорбции при $20{ }^{\circ} \mathrm{C}$ повторяет угол наклона кривой составляющей пара в смеси. Учитывая эти особенности: соотношение между влагосодержаниями при различных температурах и повтор угла наклона, авторы в своих расчетах используют кривую сорбции при $70{ }^{\circ} \mathrm{C}$, как на рис. 1, б. Авторы заранее признают неточность такой кривой сорбции, которая нуждается в дополнительной проверке. Представляет интерес определение точек кривой сорбции с помощью идентификации параметров модели. Разработанная модель 2 в стационарном расчете удовлетворяет требованиям соотношения кривой сорбции, а модель 1 - нет.

Компьютерная модель образца газобетона выполнена в программе ANSYS-FLUENT.

В табл. 1 приведены варианты решенных задач для сравнительного анализа особенностей моделей и значений их параметров, а также табличное значение коэффициента паропроницаемости для газобетона, приведенное в [1]. Значения коэффициентов, приведенные в таблице 1 , идентифицировались по результатам экспериментальных исследований.

Результаты, приведенные на рис. 2 показывают, что разработанная модель с достаточной точностью описывает процесс увлажнения блока пористого газобетона и может быть использована для моделирования теплового и влажностного состояния многослойных ограждаю-

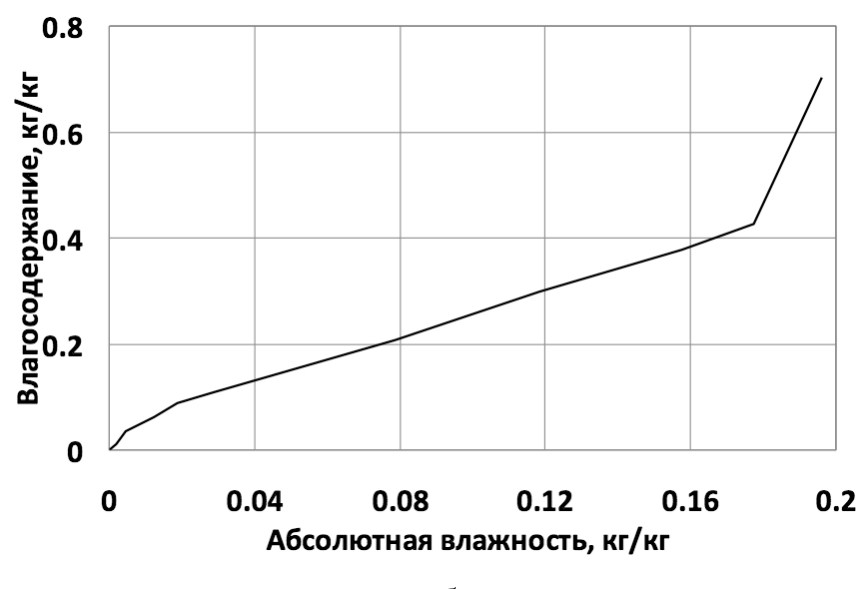

б

Рис. 1. Изотерма сорбции газобетона при температуре 20 (а) и 70 (б) ${ }^{\circ} \mathrm{C}$. 
Табл. 1. Таблица вариантов решенных задач

\begin{tabular}{|c|c|c|c|c|}
\hline Вар & Модель & $\begin{array}{c}\text { Учет последнего } \\
\text { слагаемого в } \\
\text { уравнении (6) }\end{array}$ & $\begin{array}{c}\text { Коэффициент } \\
\text { паропроницаемости } \\
\delta, \text { кг/(м·сек·Па) }\end{array}$ & $\begin{array}{c}\text { Коэффициент } \\
\text { иненсивности поглощения } \\
\text { выделения) влаги } k\end{array}$ \\
\hline & $\begin{array}{c}\text { Табличные } \\
\text { данные }\end{array}$ & Нет & $4.7 \cdot 10^{-11}$ & - \\
\hline 1 & 1 & Нет & $5 \cdot 10^{-14}$ & - \\
\hline 2 & 1 & Да & $1.5 \cdot 10^{-14}$ & $2 \cdot 10^{-14}$ \\
\hline 3 & 2 & Нет & - & $3.5 \cdot 10^{-15}$ \\
\hline 4 & 2 & Да & - & $4 \cdot 10^{-14}$ \\
\hline 5 & 2 & Да & - & \\
\hline
\end{tabular}

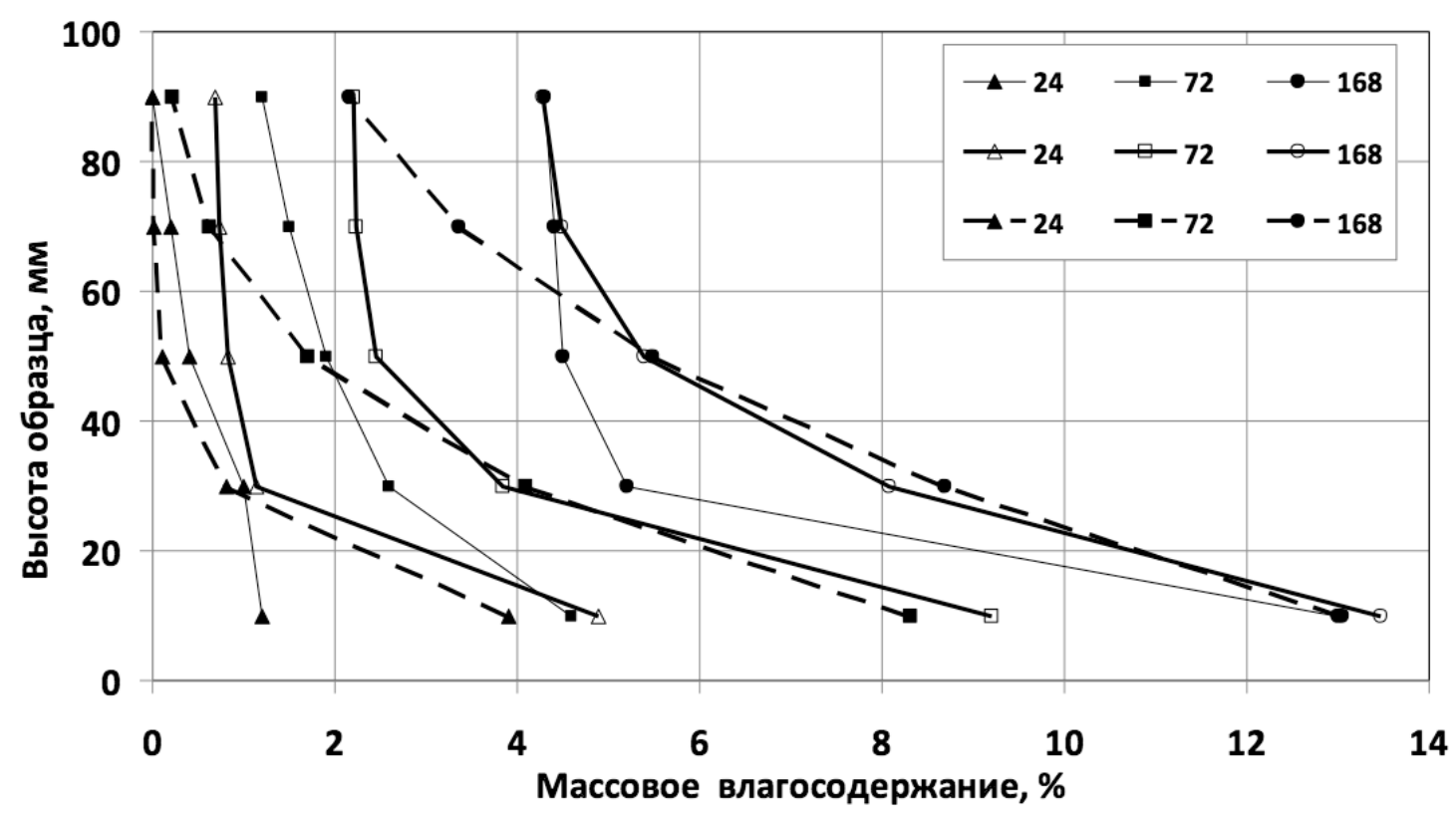

Рис. 2. Массовое влагосодержание по высоте образца газобетона в различные моменты времени. Тонкие линии - результаты экспериментальных исследований [10]; сплошные - результаты расчетов, полученные с помощью программы ANSYS-FLUENT в сопряженной постановке; прерывистые линии - в несопряженной постановке.

щих конструкций помещения. Приведенное влагосодержание на оси $x$ (рис. 2) есть сумма по массе паровой и влажностной составляющих, поскольку в эксперименте влагосодержание определялось весовым методом и гамма просвечиванием, при котором нельзя разделить общее влагосодержание на составляющие. Вариант 5 отличается от варианта 4 в табл. 1 учетом сопряженного массообмена между газобетоном и воздухом (без задания коэффициента массоотдачи от поверхности газобетона). Резкий изгиб кривых на высоте 30 мм для 72 и 168 часов можно объяснить наличием неплотностей между слоями образца по высоте, в которых могло содержаться некоторое количество воздуха.

Полученный результат был подтвержден моделированием блока газобетона в программе, описанной в [12], в модели которой идентифицировались коэффициенты паро- и влагопроницаемости в зависимости от относительной влажности и влагосодержания, соответственно. Граничные условия на границах задавались с помощью постоянного коэффициента массоотдачи, что объясняет наибольшее расхождение 
результатов на нижней и верхней границе газобетона.

Проведенные исследования позволили определить коэффициент массоотдачи, который линейно зависит от разницы концентраций на нижней границе газобетона и среды в нижней камере (рис. 3), а его величина может значительно отличаться от рекомендуемого в литературе значения $\beta=1 \cdot 10^{-8} \mathrm{\kappa} / \mathrm{m}^{2} \cdot \mathrm{c} \cdot$ Па. Сопряженная постановка позволяет в данной изотермической за- даче получить значения коэффициента массоотдачи, которые нельзя оценить в несопряженной постановке из-за отсутствия теплообмена и значения коэффициента конвективной теплоотдачи.

На рис. 4 показано соотношение паровой $m$ и влажностной $w$ составляющих по высоте газобетона. Во всех вариантах на нижней границе количество пара больше, чем влаги, поскольку в образец газобетона поступает только пар, часть которого может превратиться во

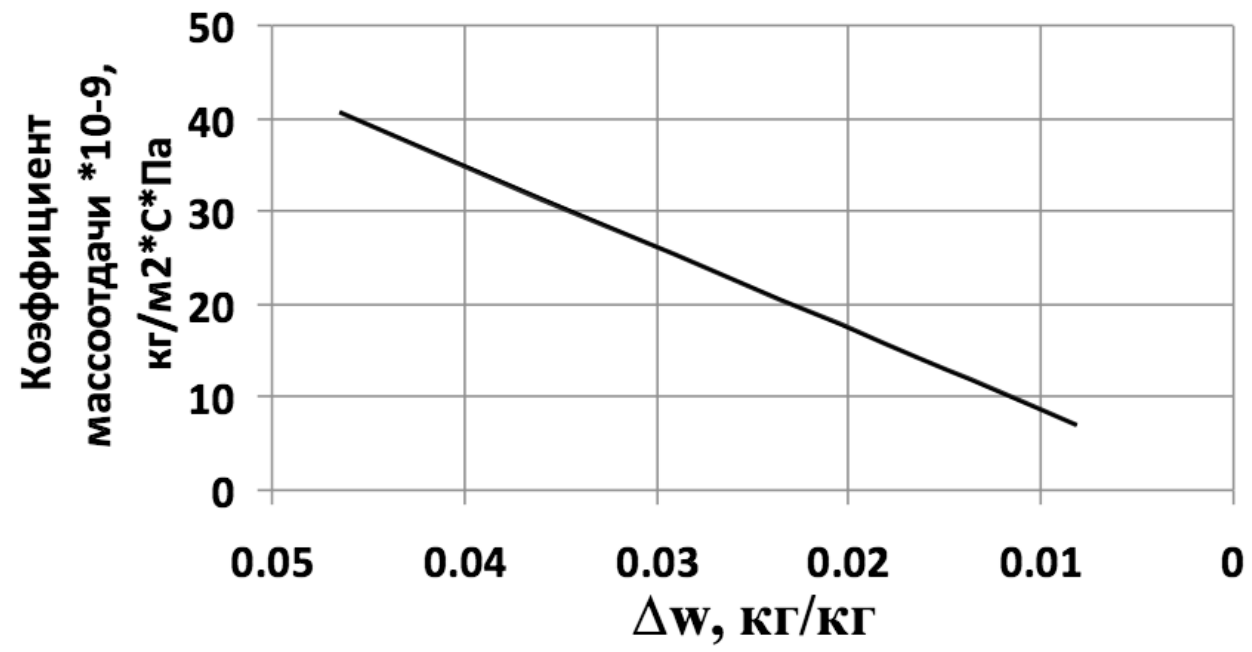

Рис. 3. Зависимость коэффициента массоотдачи от разницы концентраций влаги в нижнеей камере и на поверхности образца.

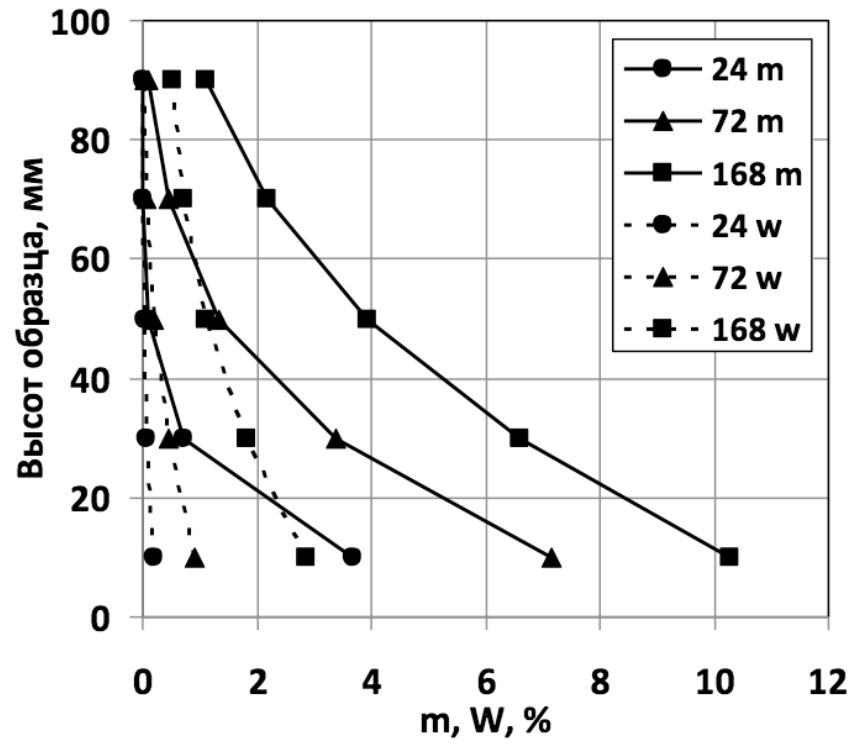

$\boldsymbol{a}$

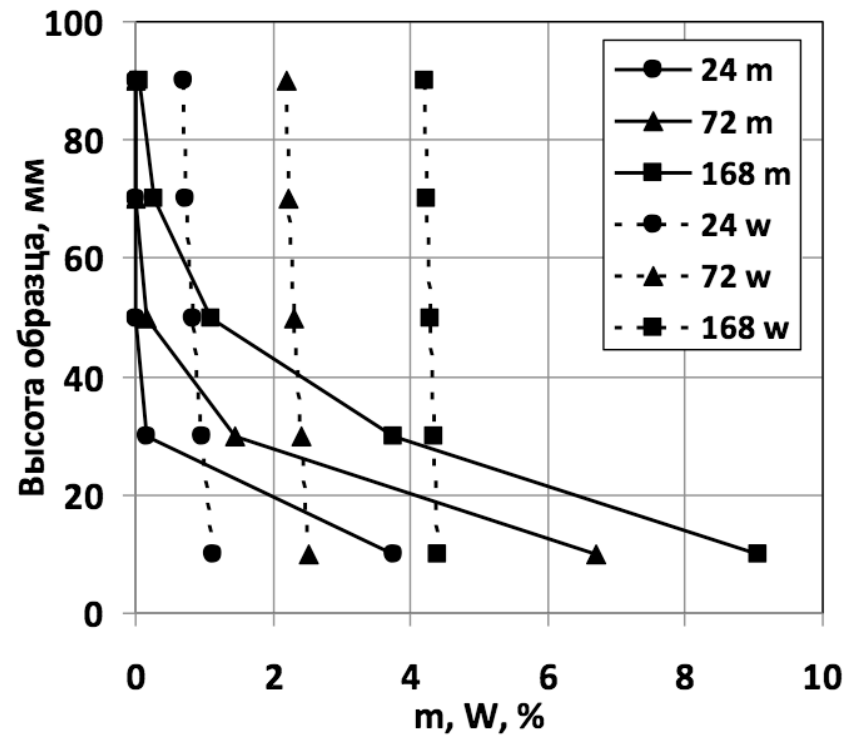

б

Рис. 4. Зависимость влагосодержсания и абсолютной влажсности по высоте образца газобетона в различные моменты времени. A-вариант 3, б-вариант 5 из табл. 1. 
влагу. Далее по высоте газобетона в варианте 3 количество пара больше, чем количество влаги, поскольку согласно уравнению (3) диффузии пара в паровоздушной смеси не удаляется часть пара, которая переходит во влагу. В варианте 5 в верхней части блока газобетона наблюдается превышение влаги над паром, поскольку насыщенная паровоздушная смесь доходит до верха образца сильно осушенной, в то время как газобетон накапливает влагу, отобранную у паровоздушной смеси, что является более физичным.

Проведенные расчеты показали, что в нестационарном режиме и при своих подобранных значениях коэффициентов $k$ и $\delta$ сумма паровой и влажностной составляющих для всех вариантов 1-5 (табл. 1) совпадают друг с другом с достаточной точностью. Результаты моделирования длительного по времени процесса увлажнения газобетона, выполненные в сопряженной постановке в программе ANSYSFLUENT, приведены на рис. 5.

Предлагаемая авторами модификация модели более физично описывает реальные про- цессы паро- и влагопереноса в строительном материале (в данном случае образце газобетона). Присутствующий пар в паровоздушной смеси может частично, как превращаться во влагу в строительном материале, так и оставаться в паровой фазе. Влага из газобетона может возвращаться в паровое состояние при наличии соответствующих условий. Т.о., в модели присутствуют и могут быть проанализированы отдельные составляющие: влага в газобетоне и пар в паровоздушной смеси в газобетоне в процессе увлажнения материала.

Модель 1 (уравнения (1) - (3)) использовать в равновесном стационарном режиме нежелательно, поскольку в ней отсутствуют предпосылки для ограничения накопления влаги в материале и выход на соотношение паровой и влажностной составляющей согласно кривой сорбции. Модель 1 также нуждается в идентификации параметров $\delta$ и $D_{w}$ по имеющимся экспериментальным данным. Для решения этой проблемы в программе [12] модифицируют и решают модель 1 относительно относительной влажности, т.е. получают

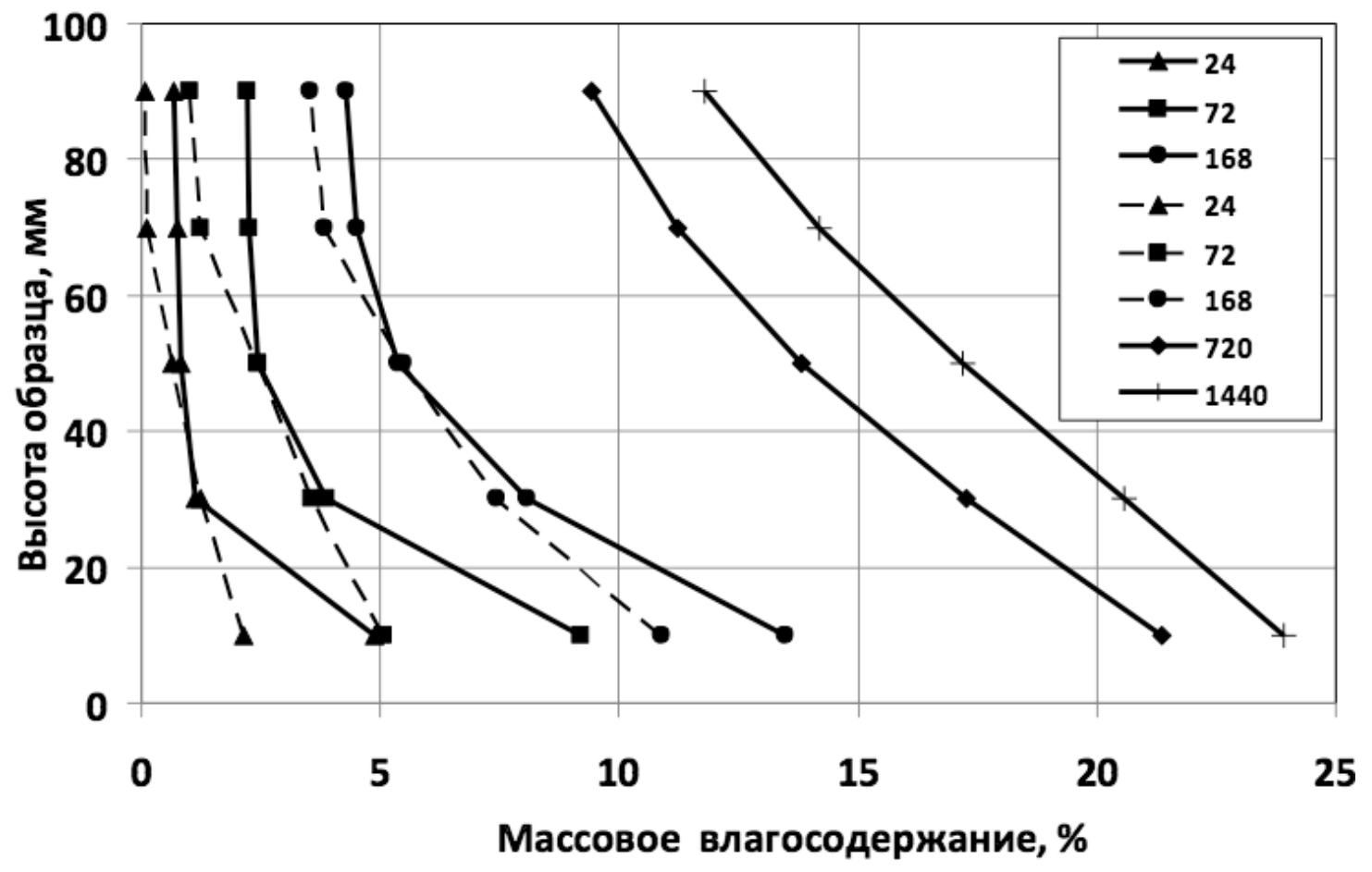

Рис. 5. Зависимость массового влагосодержания по высоте блока газобетона при длительном по времени увлажнении. 
возможность использования данных о кривой сорбции материала (в виде $\partial w / \partial \varphi)$. В таком случае модель 1 выходит на значения максимального влагосодержания отвечающего кривой сорбции. Но модель [12] не дает возможности решать сопряженную задачу, т.к. влага и пар в ней не разделяются. А для сопряженной задачи необходимо знать какое количество пара присутствует в воздухе помещения и в паровоздушной смеси ограждения, и какая его часть переходит во влагу. Разработанная авторами модель в любой момент выполняет соотношение между относительной влажностью и влагосодержанием в газобетоне согласно кривой сорбции. В нестационарном режиме обе модели 1 и 2 дают близкие результаты по распространению влаги в образце газобетона при значениях параметров $\delta$ и $k$, идентифицированных для каждой из моделей.

Специально разработанная в программной среде ANSYS-FLUENT функция, обеспечивающая решение сопряженной задачи паро- и влагопереноса, позволит также решать многомерные сопряженные задачи. Это особенно важно, т.к. накопление влаги и «точка росы» может наблюдаться в углах помещения и на стыках разных элементов конструкций и не наблюдаться на линейных элементах ограждающих конструкций.

Разработанная модель может быть использована в качестве вычислительного «стенда» для проведения вычислений с изменением любых параметров модели. Если ввести зависимость коэффициента а в формуле (5) от температуры $A f(w, m, t)$ и $D_{w}=f(t)$, модель позволит получать распределение пара и влаги для различных температур и их перепадов, как это имеет место в реальных процессах. Для практического применения иногда бывает важно рассчитывать и стационарный и квазистационарный режимы, где предложенная авторами модель подходит лучше. Также модель может позволить идентифицировать точки кривой сорбции материалов, что позволить использовать ее с большей точностью.

Дальнейшие работы в данной области будут посвящены верификации сопряженной мо- дели тепло-, паро- и влагопереноса многослойной ограждающей конструкции натурного экспериментального дома.

\section{Выводы}

1. Рассмотрена CFD- модель, учитывающая процессы сопряженного переноса пара и влаги, поступающего в пористые строительные материалы. В отличие от существующих, рассмотренная модель учитывает соотношение между паровой и влажностной составляющими в соответствии с кривой сорбции, имеющейся для рассматриваемого строительного материала.

2. Приведен анализ особенностей реализации рассмотренной модели в среде программы ANSYS-FLUENT.

3. Приведено сравнение результатов расчетов с экспериментальными данными при увлажнении газобетона. Анализ поведения кривых влаго- и паросодержания показал, что предлагаемая авторами модель более физично описывает процесс увлажнения газобетона.

Авторы статьи выражают свою благодарность д.т.н. Низовцеву М.И. из Институmа теплофизики им. С.С. Кутателадзе (Новосибирск) за консультации по вопросам экспериментальных исследований газобетона $и$ полезные советы по работе.

\section{ЛИТЕРАТУРА}

1. Фокин К.Ф. Строительная теплотехника ограждающих частей здания - М.: АВОКПРЕСС, 2006. - 256 с.

2. Богословский В.Н. Строительная теплофизика (теплофизические основы отопления, вентиляции и кондиционирования воздуха) М:. Высшая школа, 1982. - 415 с.

3. Руководство по расчету влажностного режима ограждающих конструкций зданий. М: Стройиздат, 1984. - 168 с.

4. Woloszyn M., Rode C. Tools for Performance Simulation of Heat, Air and Moisture Conditions of Whole Buildings // Building Simulation. - 2008. - Vol.1. - C. 5 - 24.

5. ANSYS FLUENT. Theory Guide. Realise 14.0. - 2011. $-862 \mathrm{c}$. 
6. Steeman H.J., Janssens A., De Paepe M. Coupling moisture transport in air flows and porous materials using CFD // Proceedings of the 8th Symposium on Building Physics in the Nordic Countries, June 16 - 18. - Copenhagen, Denmark. 2008. - P. 715 - 722.

7. Mortensen L.H., Woloszyn M., Rode C., Peuhkuri $R$. Investigation of Microclimate by CFD Modeling of Moisture Interactions between Air and Constructions // Journal of Building Physics. - 2007. - Vol. 30. - P. $279-315$.

8. Künzel H.M. Simultaneous Heat and Moisture Transfer in Building Components: Oneand Two dimensional calculation using simple parameters // Ph.D. Thesis, University of Stuttgart, Germany. -1995 . $-65 \mathrm{p}$.

9. Низовиев М.И., Стерлягов А.Н., Терехов B.И. Верификация модели расчета сопряжен-

CFD-SIMULATION OF COUPLED VAPOR AND MOISTURE TRANSFER IN POROUS BUILDING MATERIALS

Krukovsky P.G., Polubinskiy A.S., Tadlia O.Y., Kovalenko V.N.

Institute of Engineering Thermophysics of the National Academy of Sciences of Ukraine, vul. Zhelyabova, 2a, Kyiv-57, 03057, Ukraine

CFD-model is considered which takes into account the coupled transport processes separately vapor and moisture received in porous building materials. The implementation of the developed model in ANSYS-FLUENT is analyzed. Model modification proposed by the authors more physically describes the actual ного тепло- и влагопереноса при увлажнении газобетона // Известия ВУЗов. Строительство. - 2008. - №1 (589). - С. $105-110$.

10. Низовиев М.И., Стерлягов А.Н., Терехов $B . И$. Влияние градиента температуры на влагоперенос в пористых материалах // Ползуновский вестник. - 2012. - № 3/1. - С. 17 - 21.

11. ГОСТ 24816-81 Метод определения сорбционной влажности // М.: Издательство стандартов. - 1988. - 20 с.

12. Crawley D.B. Lawrie L.K., Winkelmann F.C., Buhl W.F., Huang Y.J., Pedersen C.O., Strand R.K., Liesen R.J., Fisher D.E., Witte M.J., Glazer $J$. EnergyPlus: creating a new-generation building energy simulation program // Energy and Building. - 2001. - Vol 33. - P. $319-331$.

processes of the heat and moisture transfer in the construction material (in this case - gas concrete) and the environment. Vapor in a vapor-air mixture may be partially converted into moisture in the building material or remain there in the vapor phase. Moisture from gas concrete can be returned to the vapor state in the appropriate conditions. Therefore, separate components are presented in the model and can be analyzed: moisture in the gas concrete and vapor in the vapor-air mixture in the gas concrete during humidification material. The created model may be used as computational "sample" for calculations with a change of any model parameters. It is shown a sufficient agreement between the simulation results and experimental data on wetting gas concrete.

References 12, table 1, figures 5. 
Key words: porous building materials, vapor and moisture transfer, sorption curve, CFD-modeling

1. Fokin K.F. Construction thermotechnics of the building envelope parts. // M. AVOK PRESS, 2006. $-256 \mathrm{p}$.

2. Bogoslovskiy V.N. Building thermal physics (thermal fundamentals of heating, ventilation and air conditioning) - M:. Vischaya shkola, 1982. $-415 \mathrm{p}$.

3. Manual of analysis moisture regime of building envelopes. - M: Stroyizdat, 1984. - 168 p.

4. Woloszyn M., Rode C. Tools for Performance Simulation of Heat, Air and Moisture Conditions of Whole Buildings // Building Simulation. - 2008. - Vol.1. - P. 5 - 24 .

5. ANSYS FLUENT. Theory Guide. Realise 14.0. - 2011. - 862 p.

6. Steeman H.J., Janssens A., De Paepe M. Coupling moisture transport in air flows and porous materials using CFD // Proceedings of the 8th Symposium on Building Physics in the Nordic Countries, June 16 - 18. - Copenhagen, Denmark.-2008. - P. 715 - 722 .

7. Mortensen L. H., Woloszyn M., Rode C., Peuhkuri $R$. Investigation of Microclimate by CFD Modeling of Moisture Interactions between Air and Constructions // Journal of Building Physics. - 2007. - Vol. 30. - P. 279 - 315.
8. Künzel H.M. Simultaneous Heat and Moisture Transfer in Building Components: Oneand Two dimensional calculation using simple parameters // Ph.D. Thesis, University of Stuttgart, Germany. $-1995 .-65 \mathrm{p}$.

9. Nizovtsev M.I., Sterlyagov A.N., Terekhov V.I. Verification of the model calculation of coupled heat and moisture on the gas concrete humidification // Izvestia vuzov. Stroitelstvo. - 2008. № 1 (589). - P. $105-110$.

10. Nizovtsev M.I., Sterlyagov A.N., Terekhov V.I. Effect of temperature gradient on moisture transfer in porous materials // Polzunovskiy vestnik. -2012 . - № 3/1. - P.17 - 21 .

11. GOST 24816-81 Method of determining moisture sorption // Moscow: Izdatelstvo standartov. -1988 . $-20 \mathrm{p}$.

12. Crawley D.B. Lawrie L.K., Winkelmann F.C., Buhl W.F., Huang Y.J., Pedersen C.O., Strand R.K., Liesen R.J., Fisher D.E., Witte M.J., Glazer $J$. EnergyPlus: creating a new-generation building energy simulation program // Energy and Building. - 2001. - Vol 33. - P. 319 -331.

Получено 20.11.2014 Received 20.11.2014 